\title{
Effect of the $\beta 3$-adrenergic receptor gene polymorphism Trp64Arg on BMI reduction associated with an exercise-based intervention program in Japanese middle-aged males
}

\author{
Aya Tahara $\cdot$ Yoneatsu Osaki $\cdot$ Takuji Kishimoto
}

Received: 31 January 2010/Accepted: 29 April 2010/Published online: 26 May 2010

(C) The Japanese Society for Hygiene 2010

\begin{abstract}
Objectives The $\beta 3$-Adrenergic receptor gene polymorphism Trp64Arg (ADRB3 Trp64Arg) may be associated with weight gain, especially in the East Asian populations. To date, however, the results of various investigations aimed at determining whether ADRB3 Trp64Arg has any effect on weight reduction after intervention have been inconsistent. The aim of this study was to assess the effect of ADRB3 Trp64Arg on weight reduction in a non-pharmacological intervention program.

Methods Fifty-seven Japanese men (average age $48.1 \pm$ 5.9 years) whose body mass index (BMI) was $>23.0 \mathrm{~kg} / \mathrm{m}^{2}$ participated in an intervention program in which they were encouraged to exercise by walking with a pedometer for 12 weeks. The BMI was measured at the start of the intervention program and on weeks 4,8 , and 12 (end) of the intervention period. The reduction in the BMI $(\Delta \mathrm{BMI})$ was calculated as the BMI value obtained at each point in comparison to the baseline (initial) BMI. The participants were classified into two groups: Arg allele non-carriers (Trp/Trp) and Arg allele carriers (Trp/Arg or Arg/Arg), respectively.

Results Among the participants, $61.4 \%$ were $\operatorname{Trp} / \operatorname{Trp}$ homozygous, $38.6 \%$ were Trp/Arg heterozygous, and $0.0 \%$ were Arg/Arg homozygous. There was no significant difference in the $\triangle \mathrm{BMI}$ between Arg allele non-carriers and Arg allele carriers at week 4 [non-carrier mean \pm standard deviation (SD) vs. carrier mean \pm SD: $0.25 \pm 0.43$ vs. $0.24 \pm 0.35$, respectively; $p=0.954]$, week $8(0.56 \pm 0.68$
\end{abstract}

A. Tahara $(\varangle) \cdot$ Y. Osaki $\cdot$ T. Kishimoto Division of Environmental and Preventive Medicine, Department of Social Medicine, Faculty of Medicine, Tottori University, Tottori 683-8503, Japan e-mail: bunaya@med.tottori-u.ac.jp vs. $0.58 \pm 0.61$, respectively; $p=0.913$ ), and week 12 $(0.70 \pm 0.86$ vs. $0.84 \pm 0.80$, respectively; $p=0.545)$. Conclusions Our results suggest that ADRB3 Trp64Arg does not influence BMI reduction after an exercise-based intervention program.

Keywords Exercise - Interventional study · Middle-aged men · Trp64Arg · Weight reduction

\section{Introduction}

Overweight and obese individuals have an increased risk of coronary disease, stroke, and some cancers. The number of such individuals is increasing globally [1], and in the USA these increasing numbers have led to the prediction of a shortened average life span [2]. Physical activity and improved dietary habits are effective strategies to reduce body weight, and they can also decrease the risk of associated cardiovascular disease and stroke [3]. However, there are individual differences in the effects of such intervention programs, even among individuals following the same weight reduction program. Genetic factors are associated with individual differences in weight gain [4-8], and it is possible that differences in weight reduction may also be caused by hereditary factors.

A number of different genes are believed to be associated with human obesity [9]. B3-Adrenergic receptors, which are mainly expressed in white and brown adipocytes, control lipolysis and heat production induced by noradrenaline [10]. A polymorphism resulting in a switch from tryptophan to arginine at the 64th residue in the $\beta 3$ adrenergic receptor gene, known as the ADRB3 Trp64Arg, was identified in 1995 [11-13]. The results of many subsequent studies suggest that ADRB3 Trp64Arg is 
associated with weight gain, especially in East Asian populations [14-18], thereby also leading to the possibility that ADRB3 Trp64Arg also influences weight reduction.

Several studies have compared weight reduction in individuals with the different ADRB3 Trp64Arg genotypes participating in non-pharmacological intervention programs [19-27]. Studies in Korea and the USA reported that there was no difference in weight reduction between Arg allele carriers and non-carriers [19-21]. In contrast, findings of Japanese studies have been inconsistent, with five studies showing a significant difference associated with ADRB3 Trp64Arg [22-26] and one study showing no difference [27]. It has been suggested that the differences observed in the results of the former five Japanese studies were caused by differences in the race of a subject, sex, weight at baseline, and/or the amount of weight loss by intervention. Most intervention studies have been carried out using female subjects, and there are little available data at the present time on Japanese males. Therefore, the aim of the study reported here was to examine the effect of ADRB3 Trp64Arg on weight loss associated with a nonpharmacological intervention program for Japanese male workers.

\section{Materials and methods}

\section{Subjects}

Ninety-three Japanese workers were recruited to participate in the intervention program for 12 weeks. The program was based mainly on walking. The inclusion criteria of this study were male gender, body mass index (BMI) $\geq 23.0 \mathrm{~kg} /$ $\mathrm{m}^{2}$ [28], and no past history of cardiovascular disease, cancer, or renal disease. Sixty-six men met these criteria, among whom nine were subsequently excluded because they did not provide data during business trips. Thus, data on 57 men were included in the final analysis. The average age of the subjects was $48.1 \pm 5.9$ years. The study protocol was reviewed and approved by the Ethics Committee of Tottori University School of Medicine. All of the participants provided written informed consent to participate in this study.

\section{Intervention methods}

The participants participated in an intervention program for 12 weeks during which they wore a pedometer Kenz Lifecorder EX (Suzuken, Tokyo) and started walking. The goal was 10,000 steps per day. The accumulated number of steps walked was checked once a week. The subjects received individual nutritional guidance from a dietitian at least once during the 12 -week period. They also participated in supportive group therapy aimed at providing information on arterial sclerosis and at reviewing their efforts at the initiation of their intervention program, week 6 , and at the end of the program. Both the exercise habits and eating habits before and after the intervention program were assessed by a self-administered questionnaire.

\section{Measurement of anthropometric parameters}

The subjects' height was measured at the start of the intervention. Their body weight and waist circumference were measured once a week from the start of the intervention until it was concluded. Participants wore only light clothing at the time of the body weight measurement, and the $0.5 \mathrm{~kg}$ allotted for the weight of clothes was subtracted from the value shown on the scale. The BMI was calculated as the weight (in kilograms) divided by the squared height (in square meters). The waist circumference was measured to the nearest $0.1 \mathrm{~cm}$ at the umbilicus level, with the subjects standing erect, abdomen relaxed, arms at the sides, and the feet together. The reduction $(\Delta 4 \mathrm{th}, \Delta 8$ th, $\Delta 12$ th week) was defined as: (the value after intervention - the value before intervention).

\section{Identification of ADRB3 Trp64Arg}

The Trp64Arg variation of the $\beta 3$-adrenoceptor gene was examined in peripheral blood leukocyte DNA using a TaqMan assay (Roche Diagnostics, Branchburg, NJ). The DNA was extracted using the QIAamp DNA Blood Mini Kit (Qiagen, Tokyo, Japan) according to the manufacturer's protocol. Genomic DNA was amplified using the TaqMan SNP Genotyping Assay containing the VIC probe and FAM probe (assay ID: C_2215549_20) and the TaqMan Universal PCR Master Mix containing AmpliTaq Gold DNA polymerase according to the following thermocycle profile: initial incubation steps of $10 \mathrm{~min}$ at $95^{\circ} \mathrm{C}$, followed by 40 cycles of $15 \mathrm{~s}$ at $92^{\circ} \mathrm{C}$ and $1 \mathrm{~min}$ at $60^{\circ} \mathrm{C}$. Genotyping was conducted by detecting fluorescent signals of allele-specific probes on ABI PRISM 7900HT Sequence Detection System v2.1 (Applied Biosystems, Foster, CA). The participants were notified of their genotype at the end of the intervention period upon request.

Statistical analysis

The $\chi^{2}$ test was used to determine the Hardy-Weinberg equilibrium of the genotype frequency. The participants were divided into two groups: non-carriers of the Arg allele (Trp/Trp) and carriers of the Arg allele (Trp/Arg or Arg/ Arg), respectively. Twenty-seven people in each group were necessary to estimate the indispensable sample size based on a Japanese report [27], although the study 
detected a power of $90 \%$, a significance level of $5 \%$, and a difference in BMI reduction and its standard deviation (SD) between the two groups of 0.17 and $0.19 \mathrm{~kg} / \mathrm{m}^{2}$ [29]. In addition, one group needed 34 people since the other was limited to 22 individuals. Reduction of anthropometric parameters by intervention was defined as $\Delta$ and calculated as: $\Delta 4$ th week $=($ baseline value -4 th week value $) ; \Delta 8$ th week $=($ baseline value -8 th week value $) ; \Delta 12$ th week $=$ (baseline value -12 th week value). Comparison of frequencies among category variables was performed by $\chi^{2}$ or the Fisher's exact test, where appropriate. Student's $t$ test was used to compare the difference in anthropometric parameters and its reduction between the two groups. In terms of the efficacy of the intervention, each weekly value was compared with the baseline by the paired $t$ test. Quantitative variables were expressed as the mean $\pm \mathrm{SD}$. For the purpose of adjusting for potential confounding factors, such as age, number of steps, and dietary modification during the intervention, we adopted an analysis of covariance to compare the intervention effect between the two groups. Statistical analysis was conducted using the SPSS ver. 15.0 statistical software package (SPSS, Chicago, IL). A $p<0.05$ was considered to be statistically significant.

\section{Results}

Thirty-five individuals (61.4\%) had the Trp64/Trp64 genotype (Arg allele non-carriers), while 22 (38.6\%) had the Trp64/Arg64 genotype (Arg allele carriers). No participant was a carrier of the Arg64/Arg64 genotype. The allele frequency in ADRB3 Trp64Arg was 92 for the Trp allele $(80.7 \%)$ and 22 for the Arg allele (19.3\%), which confirmed the Hardy-Weinberg equilibrium $(p=0.071)$. A comparison of characteristics between Arg allele noncarriers and carriers is shown in Table 1 . There was no significant difference between the two groups in terms of age, current smoking, everyday alcohol consumption, habitual exercisers, modifications in eating habits, medication of anti-hypercholesterolemic drugs, diabetic drugs, height, body weight, BMI, and waist circumference at baseline. There was a difference in number of steps and awareness of eating habits during the intervention between two groups. Table 2 shows the initial values at baseline and the changes in anthropometric parameters at each time point during the intervention according to ADRB3 gene variation. All anthropometric parameters, with the exception of waist circumference at week 4 , improved significantly in both groups. The reduction from baseline grew larger weekly during the intervention period in both groups. We observed no significant difference in the reduction of body weight, BMI, and waist circumference between Arg allele non-carriers and Arg allele carriers at weeks 4, 8, and 12. However, the difference in the improvement of BMI between two groups was greater in the 12th week than in the 4th and 8th weeks. After adjusting for potential confounding factors, we found no significant difference in the reduction in body weight, BMI,
Table 1 Characteristics of the participants according to variations in the Trp64Arg $\beta 3$ adrenergic receptor gene

$p$ value: $t$ test for the quantitative variables; $\chi^{2}$ test or Fisher's exact test for qualitative variables

$S D$ Standard deviation, $B M I$ body mass index

a No Arg/Arg homozygous carriers in study cohort

\begin{tabular}{llll}
\hline Parameters & $\begin{array}{l}\text { Arg allele non-carriers } \\
\text { (Trp/Trp) }\end{array}$ & $\begin{array}{l}\text { Arg allele carriers } \\
\text { (Trp/Arg) }\end{array}$ & $p$ value \\
\hline Baseline & & & \\
$\quad$ Number of participants & 35 & 22 & \\
Age, years (mean \pm SD) & $48.1 \pm 6.0$ & $48.0 \pm 5.8$ & 0.944 \\
Current smoker, $n(\%)$ & $13(37.1)$ & $4(18.2)$ & 0.150 \\
Everyday drinker, $n(\%)$ & $18(51.4)$ & $9(40.9)$ & 0.439 \\
Habitual exercisers, $n(\%)$ & $6(17.1)$ & $5(22.7)$ & 0.603 \\
Modifications in eating habits, & $19(54.3)$ & $14(63.6)$ & 0.486 \\
$n(\%)$ & $14(40.4)$ & $9(40.9)$ & 0.946 \\
Taking medicines, $n(\%)$ & $4(11.4)$ & $2(9.1)$ & 1.000 \\
$\quad$ Anti-hypercholesterolemic drugs & $1(2.9)$ & $1(4.5)$ & 1.000 \\
$\quad$ Diabetic drugs & $169.0 \pm 6.1$ & $169.3 \pm 4.9$ & 0.869 \\
Height, cm (mean \pm SD) & $76.1 \pm 10.4$ & $73.3 \pm 7.2$ & 0.279 \\
Body weight, kg (mean \pm SD) & $26.6 \pm 3.0$ & $25.6 \pm 2.3$ & 0.191 \\
BMI, kg/m ${ }^{2}$ (mean \pm SD) & $93.4 \pm 6.8$ & $91.0 \pm 5.5$ & 0.164 \\
Waist circumference, cm & & & \\
$\quad$ (mean \pm SD) & $11,732.4 \pm 3,906.6$ & $12,254.7 \pm 4,015.9$ & 0.629 \\
During intervention & $28(80.0)$ & $18(81.8)$ & 1.000 \\
Number of steps/day (mean \pm SD) & & & \\
Modifications in eating habits, $n(\%)$ & & &
\end{tabular}


Table 2 Changes in the anthropometric parameters at each time point according to variations in the Trp64Arg $\beta 3$-adrenergic receptor gene

\begin{tabular}{|c|c|c|c|c|c|c|c|}
\hline \multirow[t]{2}{*}{ Parameters/time point } & \multicolumn{2}{|c|}{ Arg allele non-carrier } & \multicolumn{2}{|c|}{ Arg allele carrier } & \multirow[t]{2}{*}{$p$ value $^{\mathrm{a}}$} & \multirow[t]{2}{*}{$p$ value $^{\mathrm{b}}$} & \multirow[t]{2}{*}{ Adjusted $R^{2 \mathrm{~b}}$} \\
\hline & Value & Reduction $(\Delta)$ & Value & Reduction $(\Delta)$ & & & \\
\hline \multicolumn{8}{|l|}{ Body weight } \\
\hline Baseline & $76.1 \pm 10.4$ & & $73.3 \pm 7.2$ & & & 0.932 & 0.276 \\
\hline Week $4 / \Delta 4$ th week & $75.3 \pm 10.5^{\mathrm{c}}$ & $0.72 \pm 1.23$ & $72.6 \pm 7.3^{\mathrm{c}}$ & $0.68 \pm 1.01$ & 0.892 & & \\
\hline Week $8 / \Delta 8$ th week & $74.5 \pm 10.6^{\mathrm{c}}$ & $1.59 \pm 1.94$ & $71.6 \pm 7.4^{\mathrm{c}}$ & $1.67 \pm 1.72$ & 0.877 & & \\
\hline Week $12 / \Delta 12$ th week & $74.1 \pm 10.6^{\mathrm{c}}$ & $1.98 \pm 2.47$ & $70.9 \pm 7.5^{\mathrm{c}}$ & $2.40 \pm 2.24$ & 0.519 & & \\
\hline \multicolumn{8}{|l|}{ BMI } \\
\hline Baseline & $26.6 \pm 3.0$ & & $25.6 \pm 2.3$ & & & 0.881 & 0.271 \\
\hline Week $4 / \Delta 4$ th week & $26.3 \pm 3.0^{\mathrm{c}}$ & $0.25 \pm 0.43$ & $25.4 \pm 2.4^{\mathrm{c}}$ & $0.24 \pm 0.35$ & 0.954 & & \\
\hline Week $8 / \Delta 8$ th week & $26.0 \pm 3.0^{\mathrm{c}}$ & $0.56 \pm 0.68$ & $25.0 \pm 2.4^{\mathrm{c}}$ & $0.58 \pm 0.61$ & 0.913 & & \\
\hline Week $12 / \Delta 12$ th week & $25.9 \pm 3.0^{\mathrm{c}}$ & $0.70 \pm 0.86$ & $24.8 \pm 2.5^{\mathrm{c}}$ & $0.84 \pm 0.80$ & 0.545 & & \\
\hline \multicolumn{8}{|l|}{ Waist circumference } \\
\hline Baseline & $93.4 \pm 6.8$ & & $91.0 \pm 5.5$ & & & 0.749 & 0.073 \\
\hline Week $4 / \Delta 4$ th week & $91.7 \pm 7.4^{\mathrm{c}}$ & $1.66 \pm 1.99$ & $89.9 \pm 5.2$ & $1.11 \pm 2.61$ & 0.376 & & \\
\hline Week $8 / \Delta 8$ th week & $91.0 \pm 7.3^{\mathrm{c}}$ & $2.34 \pm 2.73$ & $89.0 \pm 5.6^{\mathrm{c}}$ & $2.02 \pm 3.22$ & 0.690 & & \\
\hline Week $12 / \Delta 12$ th week & $90.3 \pm 7.6^{\mathrm{c}}$ & $3.13 \pm 2.89$ & $87.9 \pm 5.4^{\mathrm{c}}$ & $3.10 \pm 3.42$ & 0.968 & & \\
\hline
\end{tabular}

Data are given as the mean $\pm \mathrm{SD}$

a $p$ value with Student's $t$ test between Arg allele non-carrier and Arg allele carrier at each point

${ }^{\mathrm{b}} p$ values were calculated by analysis of covariance adjusting for age, number of steps during intervention, modifications in eating habits during intervention, and observation time

${ }^{c} p<0.01$ with the paired $t$ test between baseline and each week

and waist circumference between the two groups. The difference of the intervention effect on the anthropometric parameters between those participants on medication and those without was compared (data not shown). There was no significant difference in the values before intervention and the reduction during intervention in terms of all anthropometric parameters between the participants on anti-hypercholesterolemic or diabetic drugs and those not receiving such medications (data not shown).

\section{Discussion}

The aim of this study was to evaluate the difference in BMI reduction at 4-week intervals between Japanese middleaged men who were either Arg allele non-carriers of ADRB3 Trp64Arg or Arg allele carriers and who were participating in an intervention program. No difference was observed at weeks 4,8 , and 12 , respectively, of the intervention program between non-carriers and carriers in terms of BMI reduction from the baseline. In other words, the BMI reduction during an exercise-based intervention was not affected by the ADRB3 Trp64Arg polymorphism.

Among our subjects, the frequency of the Arg allele was $19.3 \%$, which is similar to frequencies (16.6-25.0\%) reported among Japanese in other studies [22-27].
Kuriyama et al. [27] reported that ADRB3 Trp64Arg did not affect weight reduction in a 6-month diet- and exercisebased intervention for middle-aged Japanese individuals. Although there was a difference in the sex ratio and in the average age of the participants (approximately 15 years) in the two groups (carriers and non-carriers), the results of their study are consistent with our results. In contrast, Shiwaku et al. [26] showed that Japanese peri-menopausal females who were Arg allele carriers had difficulty obtaining optimal results when participating in a weight reduction diet- and exercise-based intervention program. In their study, the BMI reduction after a 3-month intervention was approximately $0.2 \mathrm{~kg} / \mathrm{m}^{2}$, which was smaller than that at the fourth week of our study. Although the BMI had fallen by $0.8-0.9 \mathrm{~kg} / \mathrm{m}^{2}$ by the third month of our intervention, there is a possibility that a less stringent intervention, such as a $0.2 \mathrm{~kg} / \mathrm{m}^{2}$ of BMI reduction, may have revealed a difference between the Arg allele non-carriers and carriers. In their studies on Japanese obese females, Yoshida et al. [22], Sakane et al. [23], Kogure et al. [24], and Lee et al. [25] reported that Arg allele carriers had difficulty losing weight. The BMI reduction in these studies was larger, about $2 \mathrm{~kg} / \mathrm{m}^{2}$ in Arg allele non-carriers and $3 \mathrm{~kg} / \mathrm{m}^{2}$ in Arg allele carriers. Possible factors accounting for this inconsistency in the results of these studies include sex, obesity level at baseline, and the intensity of intervention. Previous BMI reduction studies 
associated with ADRB3 Trp64Arg in Korea and the USA found no association between ADRB3 Trp64Arg and weight reduction; these findings are consistent with those of our study [19-21].

Our study had a number of limitations. First, the inability to recruit a larger number of individuals for the interventional study design resulted in a relatively small sample size. If the difference in the BMI reduction between non-carriers and carriers had been smaller, then the number of study subjects was insufficient to detect an influence of ADRB3 Trp64Arg on BMI reduction. Second, based on our results, no conclusion can be reached on the effect of the homozygous Arg64 allele on weight reduction. Oizumi et al. [30] compared Trp64/Trp64 homozygous carriers with others in a cross-sectional study and showed that the BMI of homozygous Arg64/Arg64 carriers was larger than that of homozygous Trp64/Trp64, with no difference with the heterozygous Trp64/Arg64 carriers. An increased sample size would be required to examine the influence of homozygous Arg64/Arg64 on weight reduction because the frequency of this genotype is low [31]. Third, the intervention period only lasted 12 weeks. Although the reductions in the BMI at the 4th and 8th weeks between the two groups were similar, the reduction in the BMI at the 12th week in Arg allele carriers was greater (but not significantly greater) than that in Arg allele non-carriers. Therefore, if the intervention period had continued for an even longer period, then a difference according to ADRB3 polymorphism may have been detected. Therefore, future intervention studies require longer observational periods. Fourth, the influence of improved eating habits during the intervention may not have been sufficiently taken into consideration. The improvement in eating habits tended to differ from person to person, and it was difficult to control such dietary modification. Due to this situation, statistic adjustment according to dietary modification/no dietary modification during the intervention was performed.

In conclusion, we did not observe any association between the decreasing pattern of the BMI and the ADRB3 Trp64Arg during an exercise-based intervention program for middle-aged Japanese males with a BMI $>23.0 \mathrm{~kg} / \mathrm{m}^{2}$. These results suggest that ADRB3 Trp64Arg does not influence any reduction in the BMI during an exercisebased intervention program.

Acknowledgments This study was supported by a Grant-in Aid for Scientific Research (B) (term of project, 2006-2009; project number, 18390180) from the Japan Society for the Promotion of Science, Japan.

\section{References}

1. World Health Organization. Obesity and overweight. Fact sheet No. 311; 2006.
2. Stewart ST, Cutler DM, Rosen AB. Forecasting the effects of obesity and smoking on U.S. life expectancy. N Engl J Med. 2009;361:2252-60.

3. National Institutes of Health National Heart, Lung, and Blood Institute (NIHLBI). Clinical guidelines on the identification, evaluation, and treatment of overweight and obesity in adults the evidence report. NIH publication no. 98-4083. Bethesda, MD: National Institutes of Health; 1998.

4. Sorensen TI, Price RA, Stunkard AJ, Schulsinger F. Genetics of obesity in adult adoptees and their biological siblings. Br Med J. 1989;298:87-90.

5. Stunkard AJ, Sorensen TI, Hanis C, Teasdale TW, Chakraborty R, Schull WJ, et al. An adoption study of human obesity. N Engl J Med. 1986;314:193-8.

6. Stunkard AJ, Foch TT, Hrubec Z. A twin study of human obesity. J Am Med Assoc. 1986;256:51-4.

7. Stunkard AJ, Harris JR, Pedersen NL, McClearn GE. The bodymass index of twins who have been reared apart. N Engl J Med. 1990;322:1483-7.

8. Price RA, Gottesman II. Body fat in identical twins reared apart roles for genes and environment. Behav Genet. 1991;21:1-7.

9. Christopher GB, Andrew JW, Philippe F. The genetics of human obesity. Nat Rev Genet. 2005;6:221-36.

10. Emorine LJ, Marullo S, Briend-Sutren MM, Patey G, Tate K, Delavier-Klutchko $\mathrm{C}$, et al. Molecular characterization of the human beta 3-adrenergic receptor. Science. 1989;245:1118-21.

11. Walston J, Silver K, Bogardus C, Knowler WC, Celi FS, Austin $\mathrm{S}$, et al. Time of onset of non-insulin-dependent diabetes mellitus and genetic variation in the beta 3-adrenergic-receptor gene. $\mathrm{N}$ Engl J Med. 1995;333:343-7.

12. Widén E, Lehto M, Kanninen T, Walston J, Shuldiner AR, Groop LC. Association of a polymorphism in the beta 3-adrenergicreceptor gene with features of the insulin resistance syndrome in Finns. N Engl J Med. 1995;333:348-51.

13. Clément K, Vaisse C, Manning BS, Basdevant A, Guy-Grand B, Ruiz J, et al. Genetic variation in the beta 3 -adrenergic receptor and an increased capacity to gain weight in patients with morbid obesity. N Engl J Med. 1995;333:352-4.

14. Fujisawa T, Ikegami H, Kawaguchi Y, Ogihara T. Meta-analysis of the association of Trp64Arg polymorphism of beta 3-adrenergic receptor gene with body mass index. J Clin Endocrinol Metab. 1998;83:2441-4.

15. Allison DB, Heo M, Faith MS, Pietrobelli A. Meta-analysis of the association of the Trp64Arg polymorphism in the beta3 adrenergic receptor with body mass index. Int J Obes Relat Metab Disord. 1998;22:559-66.

16. Kurokawa N, Nakai K, Kameo S, Liu ZM, Satoh H. Association of BMI with the beta3-adrenergic receptor gene polymorphism in Japanese: meta-analysis. Obes Res. 2001;9:741-5.

17. Zhan S, Ho SC. Meta-analysis of the association of the Trp64Arg polymorphism in the beta3 adrenergic receptor with insulin resistance. Obes Res. 2005;13:1709-19.

18. Kurokawa N, Young EH, Oka Y, Satoh H, Wareham NJ, Sandhu MS, et al. The ADRB3 Trp64Arg variant and BMI: a metaanalysis of 44833 individuals. Int J Obes. 2008;32:1240-9.

19. Kim OY, Cho EY, Park HY, Jang Y, Lee JH. Additive effect of the mutations in the beta3-adrenoceptor gene and UCP3 gene promoter on body fat distribution and glycemic control after weight reduction in overweight subjects with CAD or metabolic syndrome. Int J Obes Relat Metab Disord. 2004;28:434-41.

20. Phares DA, Halverstadt AA, Shuldiner AR, Ferrell RE, Douglass LW, Ryan AS, et al. Association between body fat response to exercise training and multilocus ADR genotypes. Obes Res. 2004; 12:807-15.

21. Rawson ES, Nolan A, Silver K, Shuldiner AR, Poehlman ET. No effect of the Trp64Arg beta (3)-adrenoceptor gene variant on 
weight loss, body composition, or energy expenditure in obese, Caucasian postmenopausal women. Metabolism. 2002;51:801-5.

22. Yoshida T, Sakane N, Umekawa T, Sakai M, Takahashi T, Kondo M. Mutation of beta 3-adrenergic-receptor gene and response to treatment of obesity. Lancet. 1995;346:1433-4.

23. Sakane N, Yoshida T, Umekawa T, Kogure A, Takakura Y, Kondo M. Effects of Trp64Arg mutation in the beta 3-adrenergic receptor gene on weight loss, body fat distribution, glycemic control, and insulin resistance in obese type 2 diabetic patients. Diabetes Care. 1997;20:1887-90.

24. Kogure A, Yoshida T, Sakane N, Umekawa T, Takakura Y, Kondo M. Synergic effect of polymorphisms in uncoupling protein 1 and beta 3 -adrenergic receptor genes on weight loss in obese Japanese. Diabetologia. 1998;41:1399.

25. Lee JS, Kawakubo K, Inoue S, Akabayashi A. Effect of $\beta 3$-adrenergic receptor gene polymorphism on body weight change in middle-aged, overweight women. Environ Health Prev Med. 2006;11:69-74.

26. Shiwaku K, Nogi A, Anuurad E, Kitajima K, Enkhmaa B, Shimono $\mathrm{K}$, et al. Difficulty in losing weight by behavioral intervention for women with Trp64Arg polymorphism of the beta3-adrenergic receptor gene. Int J Obes Relat Metab Disord. 2003;27:1028-36.

27. Kuriyama S, Shimazu T, Hozawa A, Kure S, Kurokawa N, Kakizaki M, et al. No effect of the Trp64Arg variant of the beta3-adrenergic receptor gene on weight loss by diet and exercise intervention among Japanese adults. Metabolism. 2008; $57: 1570-5$

28. WHO Expert Consultation. Appropriate body-mass index for Asian populations and its implications for policy and intervention strategies. Lancet. 2004;363:157-63 (Erratum in: Lancet. 2004; 363:902)

29. Furukawa T, Tango T. New statistics for medicine (in Japanese). Tokyo: Asakura Publishing Co; 1993. p. 185-93.

30. Oizumi T, Daimon M, Saitoh T, Kameda W, Yamaguchi H, Ohnuma H, Funagata Diabetes Study, et al. Genotype Arg/Arg, but not Trp/Arg, of the Trp64Arg polymorphism of the beta (3)adrenergic receptor is associated with type 2 diabetes and obesity in a large Japanese sample. Diabetes Care. 2001;24:1579-83.

31. Mauriege P, Bouchard C. Trp64Arg mutation in beta 3-adrenoceptor gene of doubtful significance for obesity and insulin resistance. Lancet. 1996;348:698-9. 